\title{
Application of a Novel Venous Cannula for En-Bloc Removal of Undesirable Intravascular Material
}

\author{
Albert K. Chin, Lishan Aklog, Brian J. deGuzman and Michael Glennon \\ Vortex Medical, Inc. \\ USA
}

\section{Introduction}

Venous occlusive disease encompasses a variety of clinical entities that range the spectrum from being catastrophic and life threatening, such as massive pulmonary embolism, to disease states that may have an occult presentation, such as inferior vena cava occlusion. Other examples of veno-occlusive disease states include deep venous thrombosis and right atrial masses. When venous occlusion is characterized by an overwhelming volume of offending material, clinical therapy may be a significant challenge. This chapter examines the historical background of therapy directed at venous occlusion, and outlines a simplified technique for addressing the occurrence of major undesirable intravascular material.

\section{Etiology and incidence}

The majority of undesirable material presenting in the major venous circulation have their origins in the lower extremity veins. Deep venous thrombosis has an estimated annual incidence of over 2 million cases in the United States (Hirsh \& Hoak, 1996), and accounts for approximately 600,000 hospitalizations per year (Schreiber, 2010). The genesis of venous thrombosis continues to be aptly characterized by the observations of Virchow in 1856 (Virchow, 1998, as cited in Lopez et al., 2004), who is credited with associating the triad of (1) venous stasis, (2) endothelial injury and (3) hypercoagulability with the formation of intravascular clot. The incidence of pulmonary embolism is closely tied to the occurrence of deep venous thrombosis, so much so that the complex of deep venous thrombosis and pulmonary embolism is defined by the term "venous thromboembolism". It is estimated that approximately $50 \%$ of patients with deep venous thrombosis have detectable pulmonary emboli (Hirsh, 1996). Lower extremity deep venous thrombosis in the distal vessels, e.g. calf vein thrombosis, has commonly been held to be relatively benign, and mostly asymptomatic; however, some studies have shown that propagation of calf vein clot above the popliteal level occurs in approximately 15\% of patients (Lohr et al., 1991). Upon propagation to the popliteal vein, the risk of measurable pulmonary embolism increases to approximately 40\% (Kakkar et al., 1969, as cited in Hirsch \& Hoak, 1996).

Risk factors for venous thromboembolism are associated with conditions that alter elements of Virchow's triad. These include increasing age, surgery, trauma, hospital or nursing home 
confinement, malignancy, paralytic neurologic disease, presence of an indwelling venous catheter or pacing lead, varicose veins, previous superficial vein thrombosis, pregnancy, and oral contraceptive use (Heit, 2002). The recurrence rate for patients with a single episode of venous thromboembolism is approximately thirty percent over ten years.

The incidence of pulmonary embolism in the U.S. is estimated to be 1.35 million cases per year (Banovac et al., 2010). Predicted outcomes for patients with pulmonary embolism vary greatly with the hemodynamic stability of the patient upon presentation. Patients with a systolic arterial blood pressure below $90 \mathrm{~mm} \mathrm{Hg}$ are deemed to have massive pulmonary embolism, while patients with a systolic pressure equal to or above $90 \mathrm{~mm} \mathrm{Hg}$ are categorized as having non-massive pulmonary embolism. In the International Cooperative Pulmonary Embolism Registry involving 2,342 patients, the vast majority (95.5\%) had non-massive pulmonary embolism, while $4.5 \%$ had massive pulmonary embolism. Patients with massive pulmonary embolism had a 90 day mortality of $52.4 \%$, compared with a 90 day mortality of $14.7 \%$ in patients with non-massive pulmonary embolism (Kucher et al., 2006). In hospital mortality for patients with pulmonary embolism rose from $8.1 \%$ in clinically stable patients to $25 \%$ in unstable patients, and increased to $65 \%$ in patients requiring cardiopulmonary resuscitation, in a separate study of 1,001 patients (Kasper et al., 1997).

Inferior vena cava thrombosis is also associated with deep venous thrombosis, although to a lesser extent than the tie between deep venous thrombosis and pulmonary embolism. The frequency of IVC thrombosis in patients with deep venous thrombosis is estimated to be between 4 - 15\% (Fernandez \& Geehan, 2008). Other causes of inferior vena cava thrombosis include malignancy, trauma, surgery, abdominal aortic aneurysm, and indwelling venous catheters. In one series, carcinoma of the kidney was the most common cause of IVC thrombosis, accounting for $31 \%$ of cases presenting over a 23 year period (Siqueira-Filho et al., 1976). The actual incidence of inferior vena cava thrombosis is difficult to cite, due to the variability of its presentation. It is estimated that in patients with IVC thrombosis, over onehalf remain asymptomatic until their initial presentation with pulmonary embolism. Iatrogenic causes of inferior vena cava thrombosis are also significant. IVC thrombosis maybe a complication of vena cava filter placement, in the treatment of or prophylaxis for pulmonary embolism. The occurrence of IVC thrombosis was $2.7 \%$ in a 26 year review of 1731 patients implanted with 1765 vena cava filters (Athanasoulis et al., 2000). Vena cava thrombosis may also be caused by indwelling intravascular devices such as pacemaker leads, parenteral nutrition catheters, or hemodialysis catheters (Krug \& Zerbe, 1980; Mulvihill \& Fonkalsrud, 1984; Gouge et al., 1988).

Masses presenting in the cardiac portion of the venous circulation are relatively rare. Most of such masses are primary atrial myxomas. A study of 33108 consecutive cardiac surgical patients found an incidence of right atrial myxoma to be $0.036 \%$; this incidence represents less than $10 \%$ of all atrial myxomas, as most atrial myxomas are left sided. Thrombus formation on the tumor surface or dislodged tumor fragments may cause pulmonary embolism. Thrombus originating in the iliofemoral system or the inferior vena cava may propagate into the right atrium (Khurana \& Tak, 2004). Once clot presents in the right atrium, the prognosis is poor without active thrombectomy. A review of the literature involving twenty patients with right atrial thrombus found that the condition was uniformly fatal without treatment, while a $50 \%$ mortality rate was observed when either anticoagulation or thrombolytic therapy was administered. When surgical extraction was performed, the mortality rate was reduced to $14 \%$ (Armstrong et al., 1985). 


\section{Therapeutic history}

\subsection{Medical therapy}

Medical treatment of venous thromboembolism was instituted in 1960 with the first randomized clinical trial evaluating the efficacy of anticoagulation in patients with pulmonary embolism (Barrett \& Jordan, 1960). Anticoagulation therapy does not resolve existent thrombus, but prevents its propagation, and significantly reduces the mortality rate of pulmonary embolism. Untreated pulmonary embolism carries a mortality rate of approximately $30 \%$; this is reduced to approximately $8 \%$ when anticoagulation is instituted (Banovac et al., 2010). The typical therapeutic approach involves intravenous infusion of unfractionated or low molecular weight heparin, followed by oral anticoagulation with warfarin, which is continued for a period of several months. It has been suggested that little benefit is gained by extending anticoagulation therapy from three to six months in venous thromboembolism patients (Campbell et al., 2007).

Fibrinolytic agents provide active dissolution of clot; they were introduced into clinical treatment of venous thromboembolic disease in the 1970s (Tibbutt et al., 1974). Streptokinase, urokinase, and recombinant tissue plasminogen activator (rTPA) are compounds available for fibrinolytic therapy. All three agents convert plasminogen to plasmin, with subsequent enzymatic degradation of fibrin clot. Studies on the efficacy of the three available fibrinolytic agents demonstrate no difference in clot resolution after twentyfour hours (Almoosa, 2002). The positive effect of thrombolytic agents is partially offset by their potential for major bleeding complications, including intracerebral hemorrhage. The risk of hemorrhage during fibrinolytic therapy varies between $6-20 \%$ (Harris \& Meek, 2005). A meta-analysis of nine randomized, controlled clinical trials comparing anticoagulation alone to anticoagulation plus thrombolysis demonstrated no difference in overall mortality between the two treatment regimes in non-selected patients with acute pulmonary embolism (Thabut, G. et al., 2002). Therefore, fibrinolytic therapy is indicated for patients with massive pulmonary embolism characterized by hypotension, or potentially for normotensive pulmonary embolism patients demonstrating right heart dysfunction, as this subgroup of patients has been associated with a higher risk of mortality in previous studies (Goldhaber, 1993, as cited in Harris \& Meeks, 2005). Fibrinolytic agents may be delivered intravenously, or selectively into the pulmonary artery at the site of occlusion. A prospective, multi-center trial comparing intrapulmonary fibrinolytic infusion with intravenous fibrinolytic administration found no significant benefit with intrapulmonary catheter therapy (Verstraete et al., 1988). Instead, a prolonged intravenous thrombolytic infusion over seven hours appeared to yield a superior benefit to a single infusion over two hours. Direct insertion of an infusion catheter into the substrate of the embolus has been suggested as a more efficacious method of fibrinolytic delivery. Insufficient clinical data is available at this time to establish superiority of intra-embolic infusion over intrapulmonary or intravenous fibrinolysis.

\subsection{Surgical therapy}

Patients with massive venous thromboembolism and particularly those with contraindications to thrombolytic therapy are candidates for surgical thrombectomy and embolectomy. Pulmonary embolectomy is a substantial procedure, necessitating a median sternotomy and cardiopulmonary bypass, but without cardioplegic arrest. An arteriotomy in the main pulmonary artery is performed to allow instrumental extraction of thrombus 
under direct vision, in an en bloc fashion, if possible. In one surgical series of 29 consecutive patients, surgical pulmonary embolectomy was successful in all cases, and the resultant survival rate at one month was $89 \%$ (Aklog et al., 2002). A review of 1,300 total patients undergoing operative pulmonary embolectomy in 46 reported series from 1961 to 2005 demonstrated an average mortalityof $30 \%$ (Stein et al., 2007). Due to its degree of invasiveness, surgical embolectomy is generally reserved as the last therapeutic option.

\subsection{Catheter therapy}

Instrumentation for surgical embolectomy dates back to 1963, with the introduction of the Fogarty embolectomy balloon catheter (Fogarty, 1963). The venous thrombectomy version of the Fogarty balloon is a $6 \mathrm{~F}$ or $8 \mathrm{~F}$ catheter with inflated balloon sizes up to $19 \mathrm{~mm}$ in diameter. The venous thrombectomy catheter is primarily applied to patients with deep venous thrombosis. It incorporates a flexible distal tip to facilitate catheter passage through venous valves. Procedures are performed in the operating theater, with surgical access used to gain vascular control during the thrombectomy process.

A variety of devices have been devised for a percutaneous approach to thrombectomy. Greenfield developed a suction tip catheter for pulmonary embolectomy in 1971 (Greenfield et al., 1971). The catheter was previously available in the form of a $10 \mathrm{~F}$ steerable catheter with either a $5 \mathrm{~mm}$ or a $7 \mathrm{~mm}$ diameter cupped tip. Percutaneous entry was performed in either the femoral or jugular vein, and embolectomy conducted under fluoroscopic control. Vacuum was established by means of a syringe to aspirate a portion of the embolus into the cup, whereupon sustained vacuum was maintained as the catheter was withdrawn to remove the clot. Multiple passes of the suction cup catheter were applied until improvements in the pulmonary artery pressure and cardiac output denoted a successful clinical result. A series of 46 patients undergoing Greenfield suction pulmonary embolectomy between 1970 to 1992 demonstrated an overall success rate of $76 \%$, with a one month survival rate of $70 \%$ (Greenfield et al., 1993). Conventional straight catheter sheaths may be employed for percutaneous embolectomy. An approach termed the "Meyerovitz technique" applies vacuum via a connected 60 cc syringe to a readily available $8 \mathrm{~F}$ or $9 \mathrm{~F}$ coronary guiding catheter without distal side holes, advanced through a $10 \mathrm{~F}$ introducer sheath (Goldhaber, 1998). However, limited volumes of clot are extracted, due to the small caliber of the guiding catheters used.

Multiple catheter designs address venous thromboembolism via clot fragmentation. Peripheral angioplasty balloon catheters have been applied to restore patency in intraluminal occlusion. Dilatation of thrombus in large caliber vessels may result in significant distal embolization; therefore, balloon angioplasty may be combined with wallstent placement to decrease recurrent embolism resulting from dislodgement of balloon dilated endoluminal thrombus. Another approach to fragmentation therapy utilizes manual rotation of a pigtail catheter to disrupt clot, with concomitant site specific thrombolytic injection to augment the mechanical therapy (Schmitz-Rode et al., 1998). The 5F pigtail catheter is ensheathed in a coaxial 5.5F introducer sheath. This device seeks to recanalize an occluded vessel, without retrieval of disrupted segments. Treated patients are subjected to distal embolization that may potentially be clinically significant. Another specialized catheter fragmentation device devised by Amplatz applies high speed rotation of a distal impeller on a 7F catheter to draw clot towards the impeller, resulting in disintegration of thrombus into tiny particles. Micro thrombi generated by this system are not removed from 
the circulation. The smaller clot fragments generated by the Amplatz impeller is an improvement over pigtail embolectomy; however, the presence of circulating microhemolytic fragments may be of concern, particularly if a substantial volume of debris is generated. Clot fragmentation coupled with embolic removal has a theoretic edge over fragmentation alone. An $11 \mathrm{~F}$ Aspirex device integrating high-speed rotation of a distally situated spiral with vacuum capability is undergoing clinical trials to evaluate its effectiveness in pulmonary arteries between $6 \mathrm{~mm}$ and $14 \mathrm{~mm}$ in diameter (Kuchar, 2007). This device combination of mechanical thrombolysis with vacuum removal addresses organized thrombus and seeks the removal of fragmentation byproducts.

Another approach to clot fragmentation is catheter rheolysis. This class of therapeutic catheter utilizes a multi-lumen catheter with separate injection and retrieval ports. High pressure fluid infusion through one or more injection ports serve to fragment thrombus upon contact. Infusion through the injection port or ports create a Venturi effect, establishing a pressure differential between the injection and retrieval ports that create a backflow through the retrieval lumen for removal of thrombus fragments and infused fluid. Rheolytic catheters are sized in the 6F to 7F range, and are not designed to be used in vessels greater than $12 \mathrm{~mm}$ in diameter (Kuchar, 2007). Their application in massive pulmonary embolism has been described in small clinical series, with resultant limited effectiveness (Siablis, 2005, Chiam, 2005, Zeni, 2003, and Koning, 1997, as cited in Kuchar, 2007).

\subsection{En Bloc removal of undesirable intravascular material}

A simplified approach to the en bloc removal of undesirable intravascular material is proposed. One of the authors noticed that during surgical embolectomy, it is often possible to perform en bloc removal of a large mass of organized thrombus, when a venous drainage cannula inserted into the main pulmonary artery gains purchase of the tail of the clot. The flow rate established by the cardiopulmonary bypass pump generates significant vacuum that in some cases may pull a thrombotic cast of the pulmonary vasculature into the extracorporeal circulation, where it is trapped by the in-line filter. In other cases, the large caliber of the main pulmonary artery and the subsequent girth of the embolus prevent its introduction into the venous outflow cannula; however, it may be grasped by the cannula tip and extracted via the pulmonary arteriotomy. The mechanics of thrombectomy gained as a result of the open surgical experience suggested a possible technique of percutaneous venous extraction. An extracorporeal circuit may be established containing a large bore outflow line, a centrifugal pump, an in-line filter, and an inflow line. The distal end of the venous outflow cannula is fitted with a balloon-activated funnel tip that expands to the luminal diameter of the occluded vessel. The venous outflow cannula is advanced into proximity of the obstructing venous material, while the venous inflow cannula is inserted into a separate venous entry site. Activation of the centrifugal pump creates a unidirectional flow that serves two functions: One, it creates a vacuum at the funnel drainage side to extract undesirable intravascular material; and two it performs simultaneous reinfusion of filtered blood volume to maintain circulatory homeostasis. The extracorporeal circuit is placed percutaneously or via femoral venous cutdown. The technique establishes a venous to venous bypass circulation without oxygenation, powered by a compact centrifugal pump generating a flow rate up to 5 liters per minute. The sizes of both outflow and inflow venous cannulae are maximized, to accommodate the large girth of the pulmonary vasculature. Whereas previous suction thrombectomy devices extracted clot in piecemeal fashion with multiple catheter insertions and removals, due to their limited bore, 
the extracorporeal veno-venous system strives for en bloc extraction, with intraluminal passage of clot in the venous cannulae.

\subsubsection{Instrumentation and technique}

Elements of the funnel drainage cannula and extracorporeal circuit used to extract occluding venous material are shown in Figure 1. The funnel cannula (AngioVac ${ }^{\circledR}$ Cannula, Vortex Medical Inc., Norwell, MA) contains a $22 \mathrm{~F}$ body with distal fingers that are balloon expandable to generate a maximal funnel diameter of $48 \mathrm{~F}$. The funnel drainage cannula is connected to $1 / 2$ inch polyvinyl chloride tubing that leads to the in-line filter with a $90 \mathrm{cc}$ capacity. The centrifugal pump is downstream of the filter, and connected to the reinfusion cannula, completing the extracorporeal circuit. In preparation for establishment of extracorporeal bypass, a 10,000 unit bolus of intravenous heparin is administered to the patient, and a constant intravenous infusion of approximately 1,000 units per hour continued to maintain the patient's activated clotting time (ACT) at 300 seconds. If the ACT is maintained at 300 or above, use of a heparin coated bypass circuit is unnecessary. Prior to use, the circuit is primed with normal saline, yielding a closed system that accomplishes vacuum extraction, filtering and reinfusion in concurrent fashion. With use of the Rotaflow centrifugal pump (MAQUET Cardiovascular, Wayne, New Jersey), priming of the circuit requires approximately $500 \mathrm{cc}$ of saline. In use, venous access is established via the femoral or jugular vein, and an angiographic catheter advanced to the site of the occlusion. If extraction of pulmonary emboli in a branch vessel is anticipated, cannulation of the target vessel is performed using a $5 \mathrm{~F}$ to $8 \mathrm{~F}$ pigtail catheter (Andrews, 2004), and an 0.038" guidewire left in position. A 26F introducer sheath (Gore ${ }^{\circledR}$ DrySeal Sheath, W.L. Gore \& Associates, Inc., Medical Products Division, Flagstaff, AZ) is inserted in the femoral or jugular venous access site and the drainage cannula fitted with its internal tapered dilator is advanced over the emplaced $0.038^{\prime \prime}$ guidewire to position the funnel tip approximately 10 $\mathrm{cm}$ proximal to the occlusion. The dilator is removed from the drainage cannula, and the balloon inflated with saline to a pressure of 1 atmosphere to expand the collecting funnel. The centrifugal pump is activated prior to advancement of the funnel cannula towards the occlusion. The rotational speed of the pump is increased in increments of $500 \mathrm{ml} / \mathrm{minute}$ until thrombus extraction occurs. Flow rate indicated on the pump generally increases as the speed is increased. A sudden drop to zero flow may be a signal that a large mass has become seated in the funnel. Pump velocity is maintained, and as the mass is compressed and extracted through the cannula, flow will be re-established. If the diameter and density of a mass of dislodged organized thrombus prevents its passage through the venous drainage cannula, the cannula may be removed from the body and its lumen cleared externally. Following suction embolectomy, a completion venogram or pulmonary arteriogram is performed via the drainage cannula to evaluate the completeness of thrombus extraction.

\subsubsection{Functional mechanics of En Bloc intravascular extraction}

Intravascular extraction with the funnel cannula is a flow directed process, with the centrifugal pump generating sufficient flow to perform suction embolectomy at one end of the circuit and vascular reinfusion at the opposite end of the circuit. The balloon situated at the funnel portion of the drainage cannula not only serves to expand the funnel for vessel wall apposition and removal of intravascular material, but also provides inflow regulation during the extraction procedure (Figure 2). Modulation of antegrade venous flow assists the 


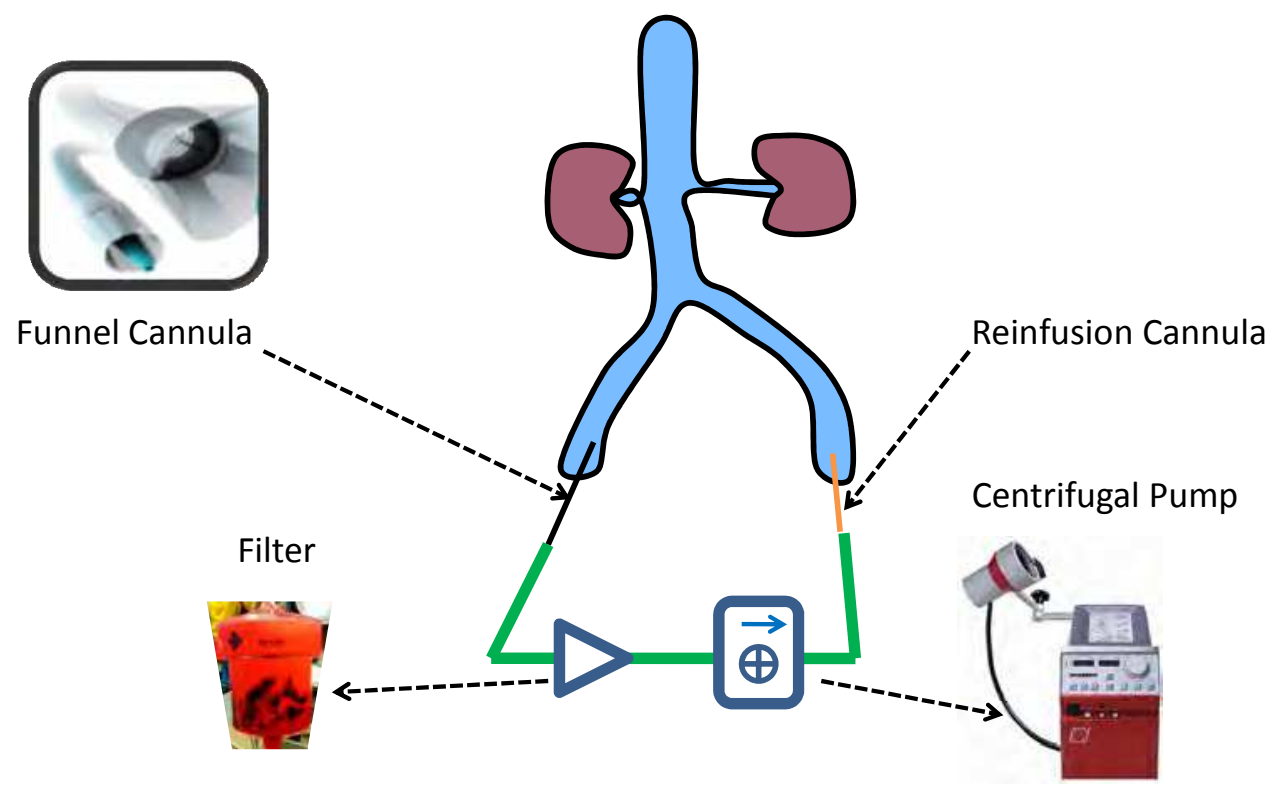

Fig. 1. Elements of the funnel drainage cannula and extracorporeal circuit

centrifugal pump in generating the necessary retrograde flow to initiate movement of the thrombotic material and its subsequent excursion the length of the drainage cannula. The degree of collateralization in the native circulation facilitates intravascular extraction, as it preserves the potential for retrograde flow at the treatment site. If retrograde flow at the occlusion site is diminished or removed, intravascular extraction may be inhibited. In order to delineate the significance of retrograde flow on the dynamics of en bloc extraction, a flow model was constructed to allow empirical evaluation of the vacuum extraction process.

\subsubsection{Material and methods}

Branched, tapering vinyl tubing was connected with fittings in a configuration that mimics the morphology of the pulmonary vasculature, and a valve was placed immediately distal to the site of embolic extraction (Figure 3). Saline was heated to 37 degrees Celsius and continuously circulated through the circuit by means of an impeller pump and a peristaltic pump in series, at a flow rate of $5.0 \mathrm{~L} /$ minute. Organized thrombus was modeled using calf liver sectioned into $15 \mathrm{~mm} \times 80 \mathrm{~mm}$ strips. Calf liver was used to model thrombus, as its density is similar to the density of mammalian clot (Nahimyak, 2006 amd Baraislas. 2007), and it lacks the propensity for disintegration in a mechanical flow model. With the thrombus model placed in the vascular circuit in a position equivalent to the main pulmonary artery, the valve proximal to the thrombus model was adjusted to three different positions: (1) completely closed position, (2) completely open position, and (3) partially closed position. The separation distance at thrombus model capture, centrifugal pump speed at capture, and flow rate generated in the funnel drainage cannula at capture were measured. 


\section{Vortex Cannula}

- Balloon Expandable Cannula

- Distal Funnel for Material Removal

- Balloon Provides Inflow Regulation

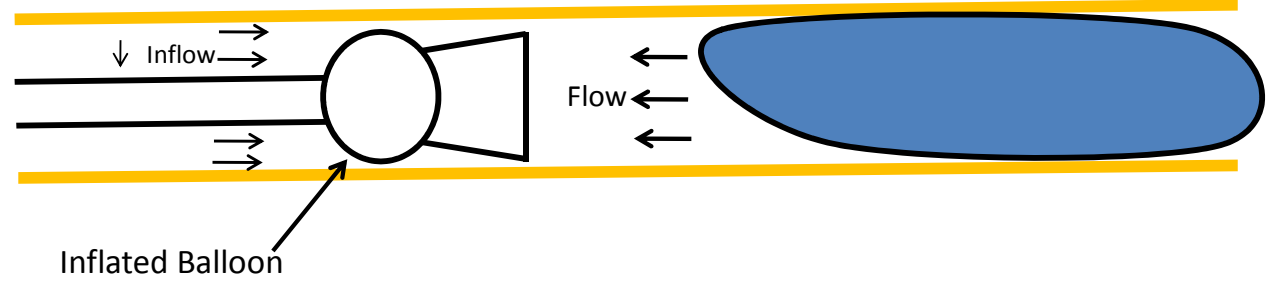

Fig. 2. Flow dynamics of the funnel drainage cannula

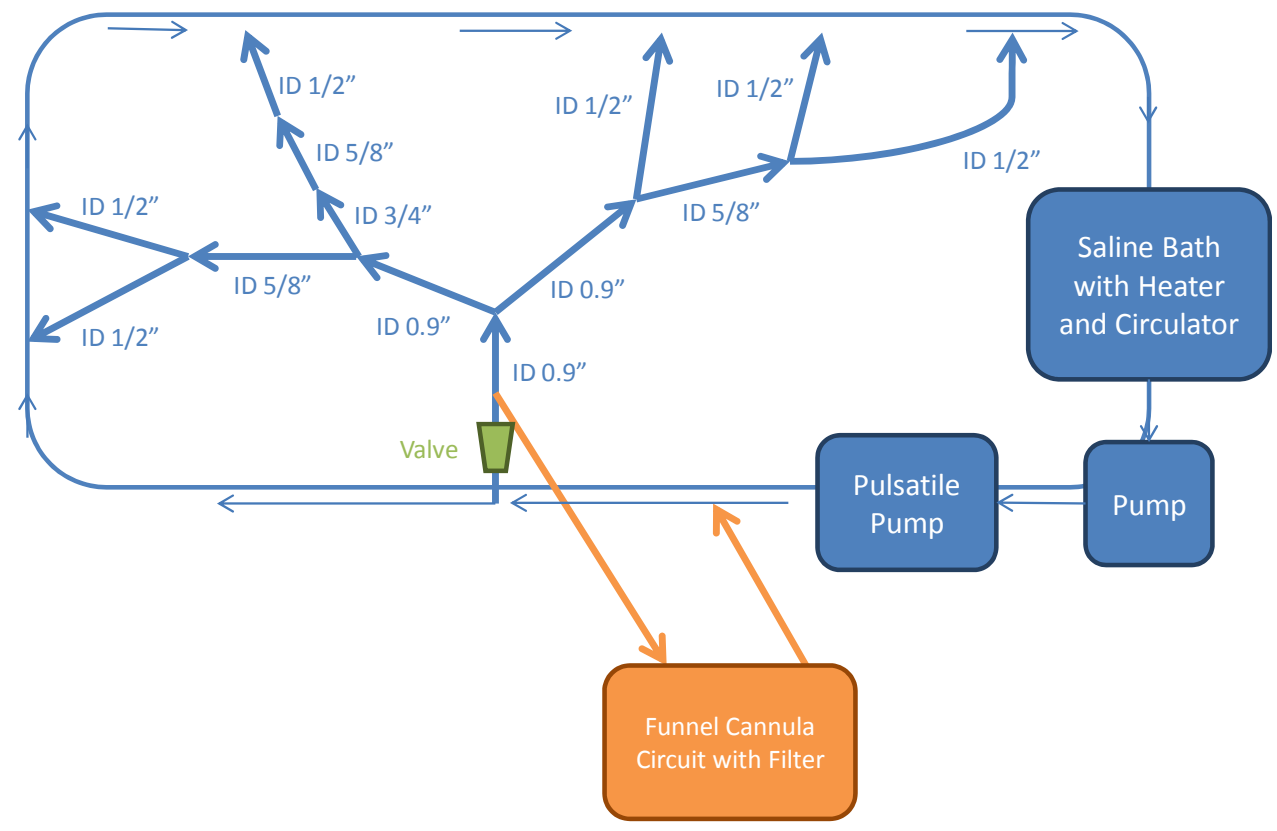

Fig. 3. Test fixture to evaluate the mechanics of the vacuum extraction process 


\subsubsection{Results}

At a separation distance of $11 \mathrm{~cm}$ between the tip of the funnel and the clot model, no movement of the clot was noted with the valve closed completely, at all pump flow rates increased to a maximum of 6 liters per minute. Advancement of the funnel cannula to the proximity of the clot model, at $5 \mathrm{~cm}$ of separation, allowed extraction at 4.6 liters per minute of pump flow rate. With the valve completely open, allowing ample backflow, the clot model was extracted at a separation distance of $11 \mathrm{~cm}$ with a pump flow rate of 2.5 liters per minute. When the valve was $80 \%$ closed, clot extraction did not occur at $11 \mathrm{~cm}$; however, removal was observed at $5 \mathrm{~cm}$ of separation distance at a pump flow rate of 6 liters per minute.

\subsubsection{Clinical experience}

Between December 2009 and May 2011, a total of 49 patients underwent removal of undesirable intravascular material using the funnel-tipped venous drainage cannula under extracorporeal veno-venous bypass. Procedures were conducted both in the fluoroscopy suite, and in the surgical theater under C-arm guidance. Veno-occlusive states addressed by these procedures included the following: (1) A retained segment of fibrin sheath from a pacemaker lead was extracted from the right atrium of a patient. (2) Vegetation was removed from the tricuspid valve of a patient who developed endocarditis from an indwelling line. (3) Thrombus and tumor was removed from a patient with an inferior vena cava occlusion due to a retroperitoneal malignancy. (4) A saddle embolus was removed from a patient with pulmonary embolism. (5) Organized and soft thrombus was removed from a patient presenting with bilateral iliofemoral and inferior vena cava occlusion. IVC thrombosis and pulmonary embolism accounted for the majority of the procedures, $43 \%$ and $29 \%$, respectively. Seven patients had right atrial thrombus removed via suction embolectomy; in four of these patients, the thrombus extended up into the superior vena cava, and cannulation of the the SVC was required to remove the occluding clot. Four patients had vegetative endocarditic masses removed from their right atria. Several representative procedures may be detailed as follows:

Example 1:

A 63 year old female presented with severe bilateral lower extremity edema, bordering on phlegmasia cerulean dolens. She had an inferior vena cava filter placed two weeks previously for deep venous thrombosis, and her history is also significant for a recent neurosurgical procedure. The patient's situation was additionally complicated by a documented history of heparin induced thrombocytopenia. A venogram demonstrated complete IVC thrombosis extending proximal to the IVC filter and involving both iliofemoral systems distally (Figure 4).

Thrombolytic therapy was contraindicated in this patient, due to the recent neurosurgical procedure and the history of heparin induced cytopenia. Rheolytic thrombectomy, applied in an attempt to recanalize the vena cava and distal venous circulation, was unsuccessful. The patient was brought back to the fluoroscopy suite, where the suction funnel cannula was inserted through a $26 \mathrm{~F}$ introducer sheath via a percutaneous right femoral vein entry site. Upon establishment of flow via the centrifugal pump, a large amount of fresh and organized thrombus was retrieved in the filter. The suction cannula was advanced into the vena cava, as well as the contralateral iliac vein. Two full filter canisters of occluding material, totaling $180 \mathrm{cc}$ in volume, was removed from the patient (Figure 5). In addition, the 


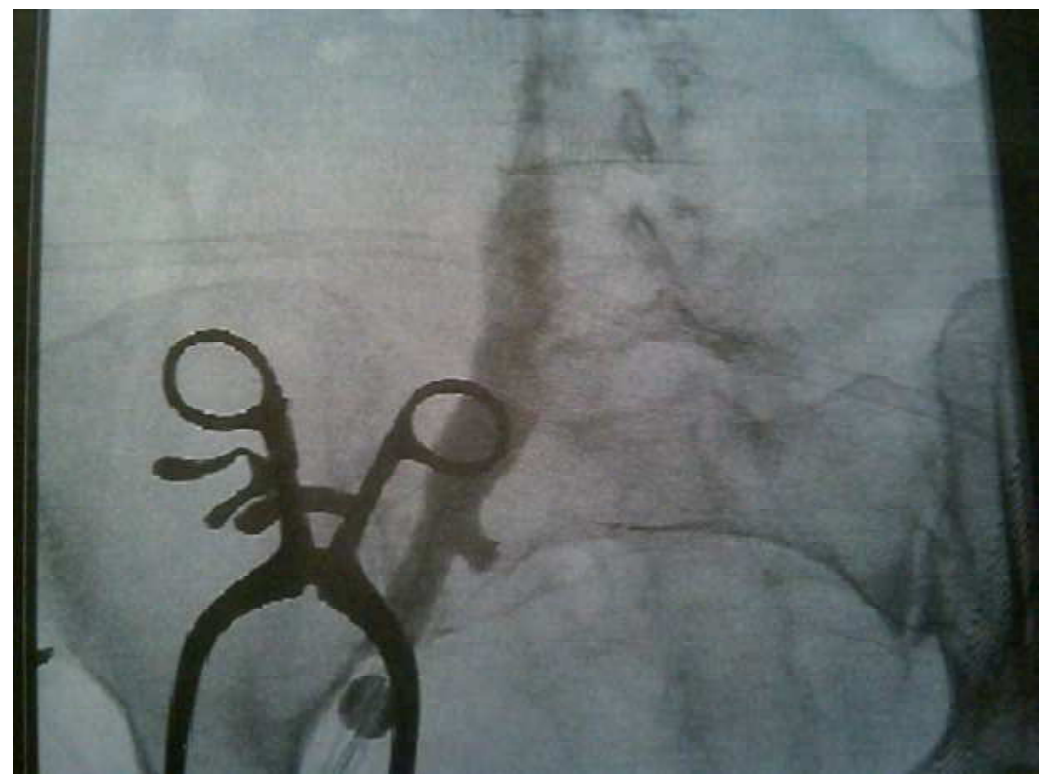

Fig. 4. Venogram depicting complete IVC thrombosis

previously placed IVC filter was removed through the central lumen of the suction cannula, while the extracorporeal circulation was maintained to prevent distal embolization from occurring during vena caval filter retrieval. Upon completion of the embolectomy procedure, a new IVC filter was placed. The post-extraction venogram showed a widely patent IVC and iliofemoral vasculature (Figure 6). The patient remained stable throughout the procedure. The procedure was conducted under administration of intravenous Bivalirudin (Angiomax ${ }^{\circledR}$, The Medicines Company, Parsippany, NJ), due to the patient history of heparin induced thrombocytopenia.

Example 2:

A 40kg 23 year old female on renal dialysis via an indwelling right subclavian catheter, with a history of end stage renal disease and a failed renal transplant, presented with chest pain. She was found to have an embolus in a distal lobar branch of the left pulmonary artery, and an echocardiogram and CT scan revealed a fluttering clot at the distal tip of the subclavian catheter, and a $3 \mathrm{~cm}$ sessile mass on the free wall of the right atrium (Figure 7). Suction embolectomy was performed under general anesthesia in the operating room under echocardiographic guidance. Due to the small stature of the patient and subsequent limited caliber of her vasculature, the left and right femoral veins were exposed via open groin incisions. The $22 \mathrm{~F}$ funnel cannula was introduced directly into the right femoral venotomy, and a 17F cannula placed into the left femoral vein for reinfusion. The funnel cannula was advanced into the right atrium and positioned in proximity to the subclavian catheter. Flow was initiated with the centrifugal pump, and chronic thrombotic material trapped by the filter. The subclavian dialysis catheter was removed, and the cannula advanced into the superior vena cava while circulating flow was maintained. A large amount of organized thrombus was noted in the filter (Figure 8). The echocardiogram showed removal of all 

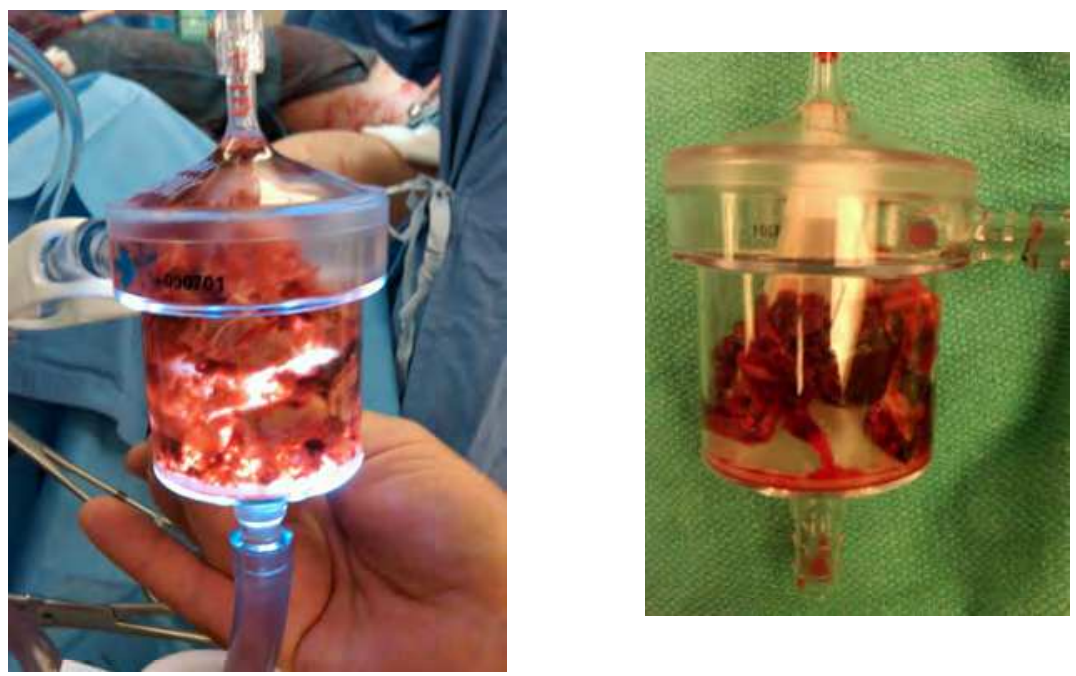

Fig. 5. Thrombotic material captured in two filters
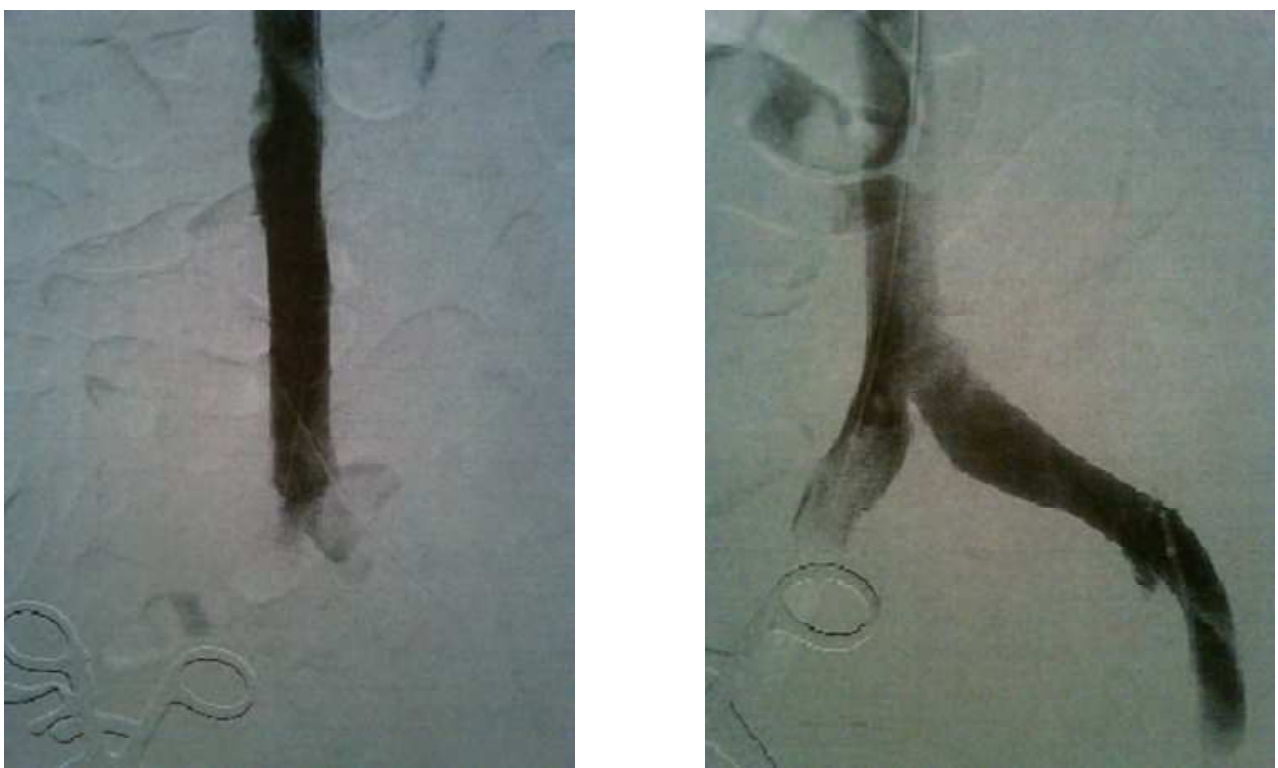

Fig. 6. Completion venogram demonstrating recanalization of the IVC and iliac veins

mobile thrombus, and a patent proximal superior vena cava (Figure 9). The sessile mass remained attached to the wall of the right atrium; no additional attempts were made to detach this immobile mass. A new dialysis catheter was placed, and the patient was discharged from the hospital under anticoagulation. 


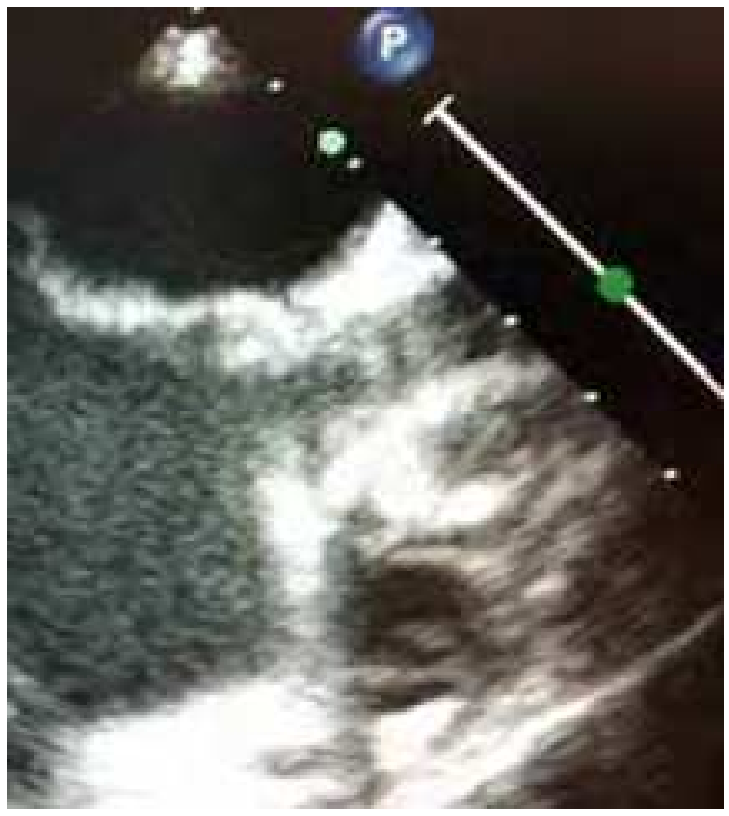

Fig. 7. Echocardiogram depicting mobile thrombus and sessile mass in the right atrium

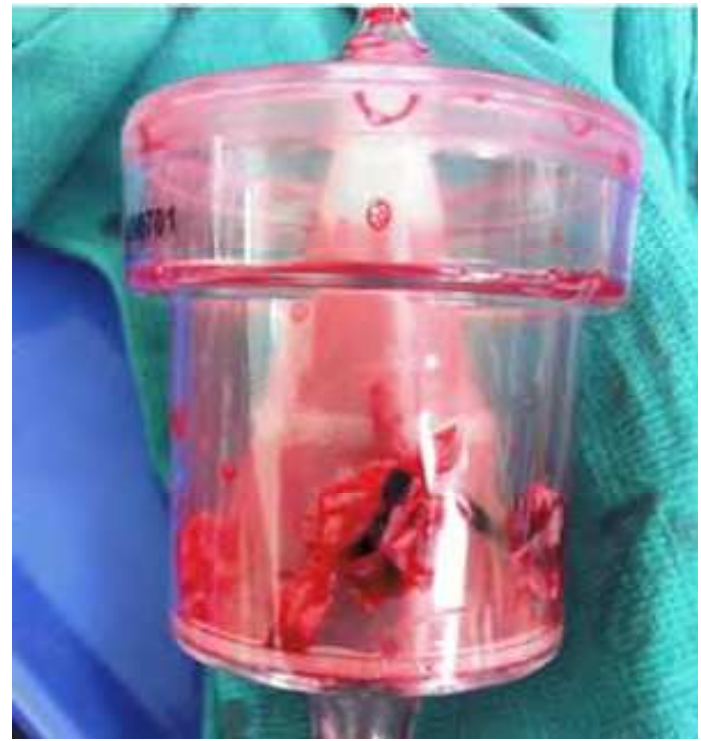

Fig. 8. Organized thrombus extracted from right atrium and superior vena cava 


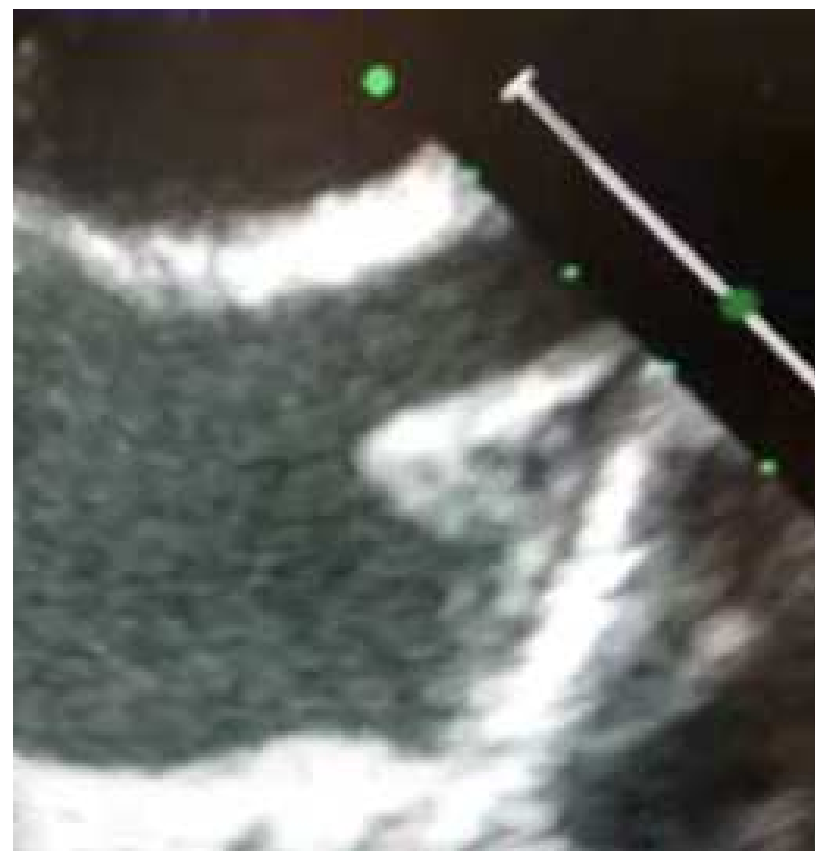

Fig. 9. Post extraction echocardiogram depicting absence of mobile thrombus

\subsubsection{Clinical results}

Of the 49 total patients in this series, 27 patients were male, and 22 female. The average age of the male patients was 53 years with a range between 27 and 88 years, and the average age of the female patients was 51 years, with a range between 19 and 82 years. Twelve of the procedures were performed in a totally percutaneous manner, while thirty-seven procedures were performed via surgical exposure of the femoral veins. Success of an extraction procedure is defined as removal of occluding material, fluoroscopic or echocardiographic evidence of venous patency, and stabilization of patient hemodynamic parameters. Forty of the forty-nine procedures resulted in removal of intravascular material for an $80 \%$ overall success rate. In 9 cases, minimal or no material was removed. In one case, spontaneous fragmentation and distal embolization of the thrombus occurred prior to initiation of the suction embolectomy procedure. One perioperative death occurred in a hemodialysis patient with a right atrial mass and an inferior vena cava occlusion. Hemothorax from a suspected guidewire perforation of the right atrium was noted during the procedure. The patient was brought to the operating room, and surgical exploration found a substantial fibrotic mass encasing the right atrium and inferior vena cava, preventing cannulation for cardiopulmonary bypass. The patient survived the surgery, but succumbed within 48 hours in the intensive care unit. No hemolysis or thrombocytopenia was observed in any of the patients in this study, and the patients' hematocrit values remained stable post procedure. 


\subsubsection{Discussion}

The propensity for clot to propagate as well as to organize renders treatment of thromboembolic disease difficult. Anticoagulation addresses further propagation of thrombus; however, once a patient presents with a significant mass of clot, vascular recanalization becomes a formidable task. Complete occlusions in major or great venous vessels tend to be unresponsive to thrombolytic therapy, as circulating thrombolytic agents are unable to access the inner mass of a substantial body of clot that typifies massive pulmonary embolism or total vena cava occlusion. Thrombolytic dissolution occurs at the periphery of the occlusion, proceeding progressively inwards with time. Hemodynamic instability may curtail the opportunity window for therapeutic intervention, and immediate bulk extraction of occlusive material is warranted. Percutaneous interventional devices utilizing mechanical or rheolytic fragmentation of clot increase the rate of thrombolysis. Dissolution is a function of the amount of interaction achieved by the device with the clot. Higher surface contact area between the active components of the device and resident clot yields greater thrombolytic activity. Presently available percutaneous devices are limited by their size relative to clot in the great vessels. The luminal cross-sectional area of a 7F catheter is equal to $3 \mathrm{~mm}^{2}$, which encompasses $0.5 \%$ of the surface area of a $30 \mathrm{~mm}$ diameter inferior vena cava or pulmonary artery with a luminal cross-sectional area of $615 \mathrm{~mm}^{2}$. This means that a 7F catheter approaching an occluding thrombus in the vena cava contacts only $0.5 \%$ of the cross-sectional area of a clot on a single pass. An impractical number of catheter passes would be required to clear a total occlusion in the great vessels.

The large bore of the funnel cannula facilitates material removal from the great vessels. The large conduit size also minimizes the potential for hemolysis during vacuum extraction. Suction therapy is conducted by a centrifugal pump which generates typical flow rates up to 5 liters per minute via the $22 \mathrm{~F}$ cannula. At this flow rate and cannula size, laminar flow is maintained in the extracorporeal circuit, providing atraumatic passage for circulating erythrocytes.

\section{Conclusion}

Removal of undesirable intravascular material using extracorporeal recirculation with a funnel venous drainage cannula seeks to mimic surgical removal of massive emboli, by maximizing physical contact with the leading edge of the occlusion, and conducting en bloc removal of substantial embolic masses. The significant flow rates (on the order of 4 or 5 liters per minute) established in the drainage cannula while extracting major emboli, are matched by simultaneous reinfusion to maintain hemodynamic stability during the embolectomy process. A funnel cannula tip that matches the size of the occluded vessel and a high circulating flow rate are necessary elements to facilitate embolectomy. Bench top tests indicate that backflow must be present to support the level of pump flow rate that generates vacuum sufficient to remove large masses. In some of the procedures that yielded little or no material extraction, it is possible that a lack of backflow was exhibited due to absent or severely limited retrograde collateralization. Guidewire or angiographic catheter passage through the thrombotic substrate may yield partial recanalization that provides the requisite degree of retrograde flow for successful embolic removal. Another cause of unsuccessful extraction may be an advanced degree of fibrotic attachment associated with aged thrombus that is not amenable to vacuum dislodgment. Further clinical experience will delineate additional associated techniques and define best patient selection criteria for optimal 
application of vacuum extraction with the funnel cannula. The experience to date generates a sense of optimism that suction embolectomy with associated extracorporeal recirculation has potential as a functional therapeutic component in the battle against thromboembolic disease. Continued research into this and other approaches is certainly warranted.

\section{References}

Aklog, L., Williams, C., Byrne, J., \& Goldhaber, S. (2002). Acute pulmonary embolectomy: A contemporary approach. Circulation, 105, (March 2002), pp. (1416-1419)

Almoosa, K. (2002). Is thrombolytic therapy effective for pulmonary embolism? Am Fam Physician, 65, (March 2002), pp. (1097-1102)

Andrews, R. (2004). Contrast peripheral phlebography and pulmonary angiography for diagnosis of thromboembolism. Circulation, 109[Suppl I], (March 2004), pp. (I-22-I-27)

Armstrong, W., Feigenbaum, H., \& Dillon, J. (1985). Echocardiographic detection of right atrial thromboembolism. Chest, 87(6), (June 1985), pp. (801-806)

Athanasoulis, C., Kaufman, J., Halpern, E., Waltman, A., Geller, S., \& Fan, C. (2000). Inferior vena caval filters: Review of a 26-year single-center clinical experience. (2000). Radiology, 216, (July 2000), pp. (54-66)

Banovac, F., Buckley D., Kuo, W., Lough D., Martin L., Millward S. Clark T., Kundu S., Rajan D., Sacks D., \& Cardella J. (2010). Reporting standards for endovascular treatment of pulmonary embolism. J Vasc Interv Radiol, 21(1), (January 2010), pp. (44-53)

Barauskas, R., Gulbinas, A., \& Baraskas, G. (2007). Investigation of radiofrequency ablation process in liver tissue by finite element modeling and experiment. Medicina (Kaunas), 43(4), (April 2007), pp. (310-325)

Barritt, D., \& Jordan, S. (1960). Anticoagulant drugs in the treatment of pulmonary embolism: a controlled trial. Lancet, 275 (7138), (June 1960), pp. (1309-1312)

Campbell, I., Bentley, D., Prescott, R., Rouytledge, P., Shetty H., \& Williamson, I. (2007). Anticoagulation for three verasus six months in patients with deep vein thrombosis or pulmonary embolism, or both: randomized trial. BMJ, 334(7595), (March 2007), pp. (674-671)

Fernandez, L., \& Geehan, D. (2008). Inferior vena cava thrombosis. In: eMedicine General Surgery, April 19, 2011, Available from http://emedicine.medscape.com/article/191103-print

Fogarty, T., Cranley, J., Krause, R., Strasser, E, \& Hafner, C. (1963). A method for extraction of arterial emboli and thrombi. Surg Gynecol Obstet, 116, (February 1963), pp. (241-244)

Goldhaber, S. (1998). Integration of catheter thrombectomy into our armamentarium to treat acute pulmonary embolism. Chest, 114, (November 1998), pp. (1237-1238)

Gouge, S., Paulson, W., \& Moore J. (1988). Inferior vena cava thrombosis due to an indwelling hemodialysis catheter. Am J Kidney Dis, 11(6), (June 1988), pp. (515-518)

Greenfield, L., Bruce, T., \& Nichols, N. (1971). Transvenous pulmonary embolectomy by catheter device. Ann Surg, 174(6), (December 1971), pp. (881-886)

Greenfield, L., Proctor, M., Williams, D., \& Wakefield, T. (1993). Long-term experience with transvenous catheter pulmonary embolectomy. J Vasc Surg, 18(3), (September 1993), pp. (450-458)

Harris, T., \& Meek, S. (2005). When should we thrombolyse patients with pulmonary embolism? A systematic review of the literature. Emerg Med J, 22(11), (November 2005), pp. (766-771) 
Heit, J. (2002) Venous thromboembolism epidemiology: implications for prevention and management. Semin Thromb Hemost, 28(Suppl 2),(June 2002), pp. (3-13)

Hirsh, J., \& Hoak, J. (1996). Management of deep vein thrombosis and pulmonary embolism. Circulation, 93, (June 1996), pp. (2212-2245)

Kasper, W., Constantinides, S., Geibel, A., Olchewski, M., Heinrich, F., Grosser, K., Rauber, K., Iversen, S., Redecker, M., \& Kienast, F. (1997). Management strategies and determinants of outcome in acute major pulmonary embolism: Results of a multicenter registry. J Am Coll Cardiol, 30, (November 1997), pp. (1165-1171)

Khurana, A., \& Tak, T. (2004). Venous thromboembolic disease presenting as inferior vena cava thrombus extending into the right atrium. Clinical Medicine $\mathcal{E}$ Research,2(2), (May 2004), pp. (125-127)

Krug, H., \& Zerbe, F. (1980). Major venous thrombosis: a complication of transvenous pacemaker electrodes. Br Heart J, 44,(August, 1980), pp.(158-61)

Kucher, N., Rossi, E.,De Rosa, M., \& Goldhaber, S. (2006). Massive pulmonary embolism. Circulation, 113(4), (January 2006), pp. (577-582)

Kuchar, N. (2007). Catheter embolectomy for acute pulmonary embolism. Chest, 132, (August 2007), pp. (657-663)

Lohr, J., Kerr T, Lutter, K., Cranley, R., Spirtoff, K, \& Cranley, J. (1991). Lower extremity calf thrombosis: To treat or not to treat? J Vasc Surg, 14(5), (November 1991), pp. (618-623)

Lopez J., Kearon C., \& Lee A. (2004). Deep venous thrombosis. Proceedings of American Society of Hematology, San Diego, California, (December 2004), pp. (439 - 464)

Mulvihill, S., \& Fonkalsrud E. (1984). Complications of superior versus inferior vena cava occlusion in infants receiving central total parenteral nutrition. J Pediatr Surg, 19(6), (December 1984), pp. (752-757)

Nahimyak, V., Yoon, S., \& Holland, C. (2006). Acousto-mechanical and thermal properties of clotted blood. J Acoust Soc Am, 119(6), (June 2006), pp. (3766-3772)

Schreiber, D. (2010). Deep venous thrombosis and thrombophlebitis. In: eMedicine Emergency Medicine, March 27, 2011, Available from:

http:/ / emedicine.medscape.com/article/1911303-overview

Siqueira-Filho, A., Kottke, B., \& Miller W. (1976). Primary inferior vena cava thrombosis. Arch Intern Med,136(7), (July 1976), pp. (799-802)

Schmitz-Rode, T., Jannsens, U., Schild, H., Basche, S., Hanrath, P., \& Gunther, R. (1998). Fragmentation of massive pulmonary embolism using a pigtail rotation catheter. Chest, 114, (November 1998), pp. (1427-1436)

Stein, P., Alnas, M., Beemath, A., \& Patel, N. (2007). Outcome of pulmonary embolectomy. Am J Cardiol, 99(3), (February 2007), pp. (421-423)

Thabut, G., Thabut, D., Myers, R., Bernard-Chabert, B., Marrash-Chahla, R., Mal, H., \& Fournier, M. (2002). Thrombolytic therapy of pulmonary embolism. JACC, 40(9), (November 2002), pp. (1660-1667)

Tibbutt, D., Davies J., Anderson, J., Fletcher, E., Hamill, J., Holt, J., Thomas, M., Lee, G., Miller G., Sharp, A., \& Sutton, G. (1974). Comparison by controlled clinical trial of streptokinase and heparin in treatment of life-threatening pulmonary embolism. $B M J, 1$, (March 1974), pp. (343-347)

Verstraete, M., Miller, G., Bounameaux, H., Charbonnier, B., Colle, J., Lecorf, G., Marbet, G., Mombaerts, P., \& Olsson, C. (1988). Intravenous and intrapulmonary recombinant tissue-type plasminogen activator in the treatment of acute massive pulmonary embolism. Circulation, 77(2), (February 1988), pp. (353-360) 


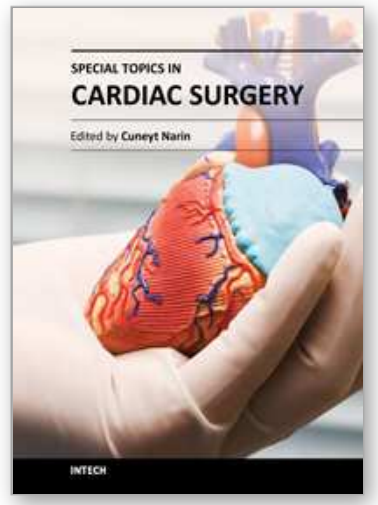

\author{
Special Topics in Cardiac Surgery \\ Edited by Prof. Cuneyt Narin
}

ISBN 978-953-51-0148-2

Hard cover, 308 pages

Publisher InTech

Published online 29, February, 2012

Published in print edition February, 2012

This book considers mainly the current perioperative care, as well as progresses in new cardiac surgery technologies. Perioperative strategies and new technologies in the field of cardiac surgery will continue to contribute to improvements in postoperative outcomes and enable the cardiac surgical society to optimize surgical procedures. This book should prove to be a useful reference for trainees, senior surgeons and nurses in cardiac surgery, as well as anesthesiologists, perfusionists, and all the related health care workers who are involved in taking care of patients with heart disease which require surgical therapy. I hope these internationally cumulative and diligent efforts will provide patients undergoing cardiac surgery with meticulous perioperative care methods.

\title{
How to reference
}

In order to correctly reference this scholarly work, feel free to copy and paste the following:

Albert K. Chin, Lishan Aklog, Brian J. deGuzman and Michael Glennon (2012). Application of a Novel Venous Cannula for En-Bloc Removal of Undesirable Intravascular Material, Special Topics in Cardiac Surgery, Prof. Cuneyt Narin (Ed.), ISBN: 978-953-51-0148-2, InTech, Available from: http://www.intechopen.com/books/special-topics-in-cardiac-surgery/application-of-a-novel-venous-drainagecannula-for-en-bloc-removal-of-undesirable-intravascular-mate

\section{INTECH}

open science | open minds

\author{
InTech Europe \\ University Campus STeP Ri \\ Slavka Krautzeka 83/A \\ 51000 Rijeka, Croatia \\ Phone: +385 (51) 770447 \\ Fax: +385 (51) 686166 \\ www.intechopen.com
}

\author{
InTech China \\ Unit 405, Office Block, Hotel Equatorial Shanghai \\ No.65, Yan An Road (West), Shanghai, 200040, China \\ 中国上海市延安西路65号上海国际贵都大饭店办公楼 405 单元 \\ Phone: +86-21-62489820 \\ Fax: +86-21-62489821
}


(C) 2012 The Author(s). Licensee IntechOpen. This is an open access article distributed under the terms of the Creative Commons Attribution 3.0 License, which permits unrestricted use, distribution, and reproduction in any medium, provided the original work is properly cited. 